\title{
ANÁLISIS COMPARATIVO DE LOS MÉTODOS DE EULER Y RUNGE-KUTTA EN LA SOLUCIÓN NUMÉRICA DE ECUACIONES DIFERENCIALES DE PRIMER ORDEN MEDIANTE PROGRAMACIÓN EN MATHCAD
}

\author{
Euler's and runge-kutta of approximate solution of first-order \\ differential equations. iterative methods for mathcad
}

CARLOS M. MATA RODRÍGUEZ*

Recibido: 19 de Octubre de 2015. Aceptado: 19 de Noviembre de 2015

DOI: http://dx.doi.org/10.21017/rimci.2016.v3.n5.a2

\begin{abstract}
Resumen
El surgimiento de la teoría de las ecuaciones diferenciales ordinarias, data de finales del siglo XVII. En un primer intento se crearon procedimientos independientes, para resolverlas, pero resulto claro que un gran número de estas ecuaciones en el acto de hallar su solución no correspondían con los métodos clásicos, esto es, expresarlas por medio de funciones elementales del Cálculo por lo que no podían ser resueltas. No fue hasta el siglo XIX que los matemáticos se dieron cuenta que solo un número relativamente pequeño de ecuaciones diferenciales podía resolverse aplicando funciones elementales. En temprana fecha, uno de los primeros que se percato de tal cuestión fue el matemático de origen suizo Leonardo Euler que en el año de 1768 desarrollo el primer método numérico para la solución de las ecuaciones diferenciales, posteriormente se han desarrollado varios que en su forma general siguen la línea dejada por Euler, hasta llegar a uno de gran precisión e intenso uso que es el método iterativo de RungeKutta. El presente trabajo presenta un análisis de ambos métodos, desarrollando sus algoritmos básicos, programados en Mathcad. Pudiéndose comprobar al finalizar el grado de exactitud que presenta cada uno en la solución numérica de las ecuaciones diferenciales.
\end{abstract}

Palabras clave: ecuaciones diferenciales, métodos numéricos, programación estructurada, procesos iterativos.

\begin{abstract}
The emergence of the theory of ordinary differential equations, data from the late seventeenth century. In a first attempt to separate procedures were created to resolve, but it was clear that a large number of these equations in the act of finding its solution did not correspond to the classical methods, this is, express them through elementary functions of calculus so they could not be resolved. It was not until the nineteenth century that mathematicians realized that only a relatively small number of differential equations could be solved by applying elementary functions. In early date, one of the first who noticed this question was the mathematician Swiss-born Leonardo Euler that in 1768 developed the first numerical method for solving differential equations, subsequently developed several that in form generally follow the line left by Euler, until one of great precision and intense use is the iterative Runge-Kutta methods. This paper presents an analysis of both methods, developing their basic algorithms, programmed in Mathcad. Being able to check at the end of the degree of accuracy that has everyone in the numerical solution of differential equations.
\end{abstract}

Keywords: differential equations, numerical methods, structured programming, iterative processes.

* Profesor Licenciado en Matemáticas. Consultor para la Formación de Personal en Informática. Miembro de la ANIR (Asociación Nacional de Inventores y Racionalizadores). Actualmente Departamento de Matemáticas, Universidad de Ciego de Ávila. Cuba. Correo electrónico: camaro@unica.cu 


\section{INTRODUCCIÓN}

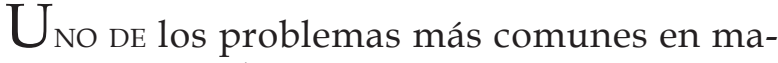
temáticas es resolver una ecuación, pero si se trata de una ecuación diferencial la dificultad se acrecienta en gran medida[1-3].

Las ecuaciones que involucran la derivada de una función de una sola variable, ocurren prácticamente en todas las ramas de las matemáticas [1][2]. En su forma general, toda situación que se refiere a la rapidez de variación de una variable con respecto a otra conduce a una ecuación diferencial.

Los conocedores del tema saben que existen muchas técnicas y procedimientos para encontrar la solución a ecuaciones diferenciales en términos de funciones elementales[4][5], tales como polinomios, funciones trigonométricas, exponenciales y logarítmicas, pero es necesario destacar que a menudo los problemas que se presentan en la práctica o bien no pueden resolverse por los métodos clásicos, o la solución es tan difícil de obtener que el cálculo sobrepasa nuestras posibilidades algebraicas[6].

El problema fundamental consiste en que la ecuación ha sido definida, por ende se debe hallar su solución. Se entiende por solución una función que exprese la relación que debe existir entre las variables de tal modo que la ecuación diferencial sea satisfecha [3].

En gran número de aplicaciones prácticas determinados coeficientes o funciones de las ecuaciones diferenciales tienen una marcada tendencia a la no linealidad, o están representados en forma de un conjunto de datos tabulados obtenidos experimentalmente, lo cual elimina de inmediato las posibilidades de obtener una solución por los métodos tradicionales[7][8].

Por las razones expuestas, a lo largo de la historia de las ecuaciones diferenciales los matemáticos han desarrollado procedimientos para hallar las soluciones donde los métodos clásicos no resultan ser útiles.

\section{Desarrollo}

El objetivo fundamental del presente artículo consiste en establecer una comparación entre dos métodos numéricos para la solución de la ecuación diferencial.

$$
\frac{d y}{d x}=y^{\prime}=f(x) \text { Siendo } y=y_{o} \text { cuando } x=x_{o}
$$

Se trataran dos procedimientos clásicos: El método de Euler [9] (el primero cronológicamente) y el de Runge-Kutta [9] (de amplia divulgación) programados en Mathcad mediante un ejemplo demostrativo.

Es necesario destacar que los procedimientos indicados requieren un largo y tedioso cálculo para llegar a la solución numérica de la ecuación diferencial, obteniendo en la mayoría de los casos sólo modestas aproximaciones, no fue hasta la aparición de los dispositivos electrónicos de cálculo [5] [6] que estos métodos pudieron ser desarrollados con toda efectividad.

En la década de los años 30 el estadounidense Vannevar Bush diseñó el analizador diferencial para la resolución de las ecuaciones diferenciales el cual sentó las bases que permitirían el desarrollo de la primera computadora digital, el Mark I[10]

Para comenzar sin más dilación la esencia del presente trabajo vamos a calcular la solución numérica de la ecuación diferencial:

$$
\frac{d y}{d x}=1+\frac{\left(x+x^{2}\right)}{e^{y}}
$$

Con las condiciones iniciales $\mathrm{y}(0)=0 \mathrm{y}$ calcularemos y para $x=0.6$.

Como comprobación de los resultados, para $x=\underline{0.6}$

$\mathrm{Y}=0.75540586$ con ocho cifras decimales, dicho valor fue calculado con la computadora IBM 360/67 según la publicación de la revista American Mathematical Computer de marzo de 1962 (comprobado con MAPLE).

\section{El MÉTOdo DE Euler (LeONARD EULER, 1707-1783, SUIZA)}

La complejidad del problema de resolución de las ecuaciones diferenciales y la imposibilidad de

Rev. Ingeniería, Matemáticas y Ciencias de la Información Vol. 3 / Núm. 5 / enero-junio de 2016; pág. 23-27 
integrarla sirvió de causa fundamental para el surgimiento del cálculo numérico en la solución de ecuaciones diferenciales. De ello se percato Euler y sus colaboradores y el método del cual hemos hecho referencia se publicó en 1768, en el tomo III de su célebre Cálculo Integral [10].

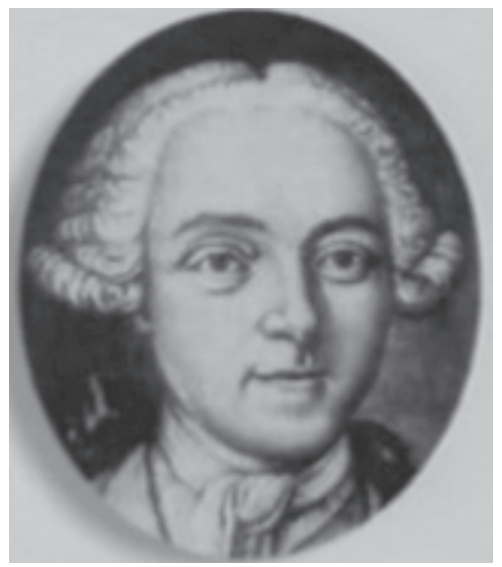

Fig. 1. Leonard Euler.

El método tiene por fundamentación la aproximación de una curva mediante una secuencia de líneas rectas, lo que determina errores de cálculo desde un principio. Este método requiere utilizar valores muy pequeños en el incremento para lograr una aceptable aproximación de la solución de la ecuación [3].

A medida que nos alejamos del valor inicial, la solución aproximada pierde precisión [8] (se aleja de la solución exacta), Cuando se reduce el incremento, la solución mejora, pero las iteraciones crecen es forma exponencial.

Esto trae como consecuencia la enorme cantidad de cálculos a ejecutar. Este método es comparativamente muy aproximado y se emplea en lo fundamental, para los cálculos preliminares. Sin embargo los fundamentos del método sirvieron de partida para lograr aproximaciones mas precisas [11].

Después del fallecimiento de Euler sus discípulos perfeccionaron el método, conocido como Método de Euler Mejorado [10], (Este método se basa en la misma idea del método anterior, pero hace un refinamiento en la aproximación, tomando un promedio entre las pendientes) incorporando procedimientos algebraicos que mejoraban parcialmente la solución.
Veamos ahora el programa de cálculo en Mathcad [12][13].

Definimos la ecuación diferencial:

$$
f(x, y)=1+\frac{\left(x+x^{2}\right)}{e^{y}}
$$

Fundamentación analítica:

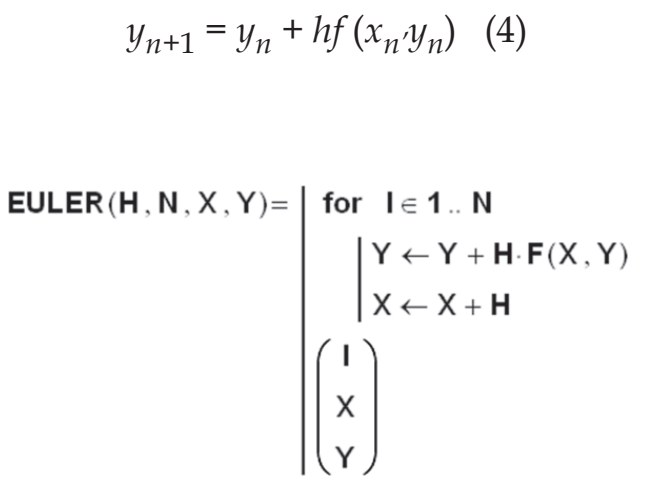

Fig. 2. Programa en Mathcad.

El proceso que lleva a efecto el cálculo consta de cuatro parámetros: $\mathrm{H}, \mathrm{N}, \mathrm{X}, \mathrm{Y}$.

1) H representa el valor incremental.

2) $\mathrm{N}$ el número de iteraciones.

3) $\mathrm{X}$ e $\mathrm{Y}$ los valores con las condiciones iniciales.

Los resultados se muestran en la Fig. 3.

$$
\operatorname{EULER}(0.1,6,0,0)=\left(\begin{array}{c}
6 \\
0.6 \\
0.73435843
\end{array}\right)
$$

Fig. 3. Resultados en forma matricial con 6 iteraciones.

La solución es: 0.73435843

Con 6 iteraciones se logra un error relativo porcentual de $2.79 \%$ comparando con la solución antes indicada. (IBM/360) [11].

Si se aumenta el número de iteraciones (60) y se reduce el incremento (0.01), el resultado es: 


$$
\operatorname{EULER}(0.01,60,0,0)=\left(\begin{array}{c}
60 \\
0.6 \\
0.75338083
\end{array}\right)
$$

Fig. 4. Resultados en forma matricial con 60 iteraciones.

Para un error relativo de $0.27 \%$.

Para finalizar, con un incremento de 0.0001 , el número de iteraciones es 6000, y el error porcentual $=0.00267$.

$$
\operatorname{EULER}(0.0001,6000,0,0)=\left(\begin{array}{c}
6000 \\
0.6 \\
0.75538569
\end{array}\right)
$$

Fig. 5. Resultados en forma matricial con 6000 iteraciones.

\section{El MÉTOdo De Runge-KutTA}

El método que se expone fue ideado por Carl Runge (1856-1927, Alemania) hacia 1894, y ampliado por Martin Kutta (1867-1944, Alemania) años después, Es un método de precisión elevada. Tiene características similares al de Euler [10].
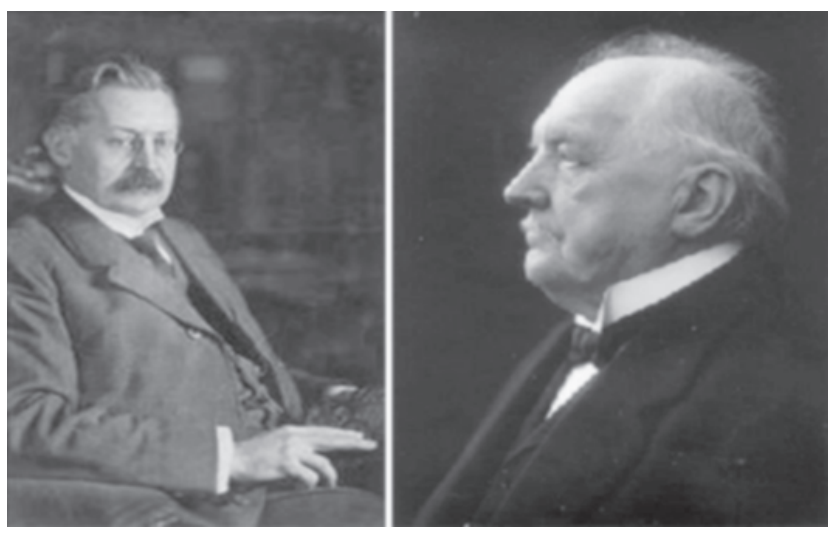

Fig. 6. A la Izquierda Carl Runge y a la derecha Martin Kutta.

El algoritmo para el método de Runge-Kutta de cuarto orden, o método clásico de Runge-Kutta, abreviado como RK4, es de uso extendido, y reconocido como una valiosa herramienta de cálculo, por la buena aproximación que produce [5].

El procedimiento, aparece dentro del contexto del programa diseñado en Mathcad.

Tiene la ventaja, sobre otros métodos tales como el de Picard, Taylor y Milne que no re- quiere de complejas operaciones de integración y derivación [7].

Se resolverá la ecuación diferencial propuesta y al finalizar se realiza un análisis comparativo de los resultados obtenidos.

$$
f(x, y)=1+\frac{\left(x+x^{2}\right)}{e^{y}}
$$

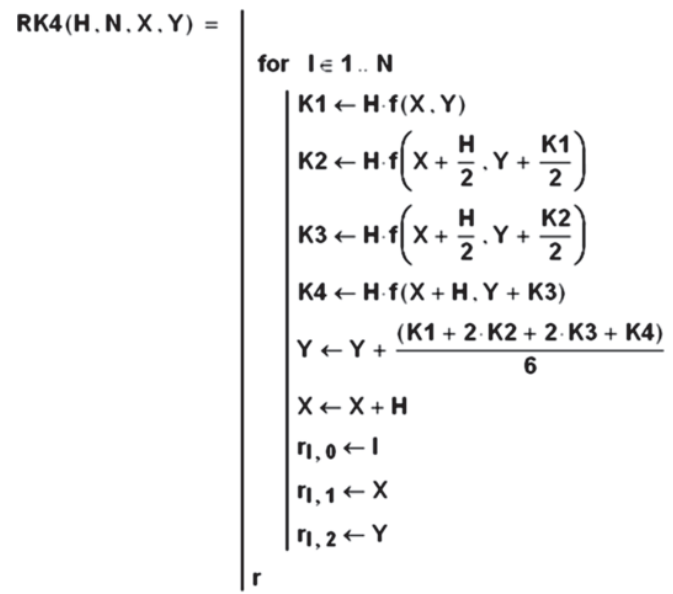

Fig. 7. Programa en Mathcad.

La solución ha sido expresa en forma de tabla matricial, como se observa en la Fig. 8, que sólo han sido necesarias 6 iteraciones para obtener una solución de alta precisión.

$\operatorname{RK4}(0.1,6,0,0)=\left(\begin{array}{lll}I & X & Y \\ 1 & 0.1 & 0.10497578 \\ 2 & 0.2 & 0.21962626 \\ 3 & 0.3 & 0.34318918 \\ 4 & 0.4 & 0.47455513 \\ 5 & 0.5 & 0.61241714 \\ 6 & 0.6 & 0.75540602\end{array}\right)$

Fig. 8. Resultados en forma matricial con 6 iteraciones.

Comparando con las 6000 iteraciones del método de Euler la evidencia no requiere más comentarios.

Esto demuestra el por qué es tan difundido el método de Runge-Kutta en la literatura matemática. 


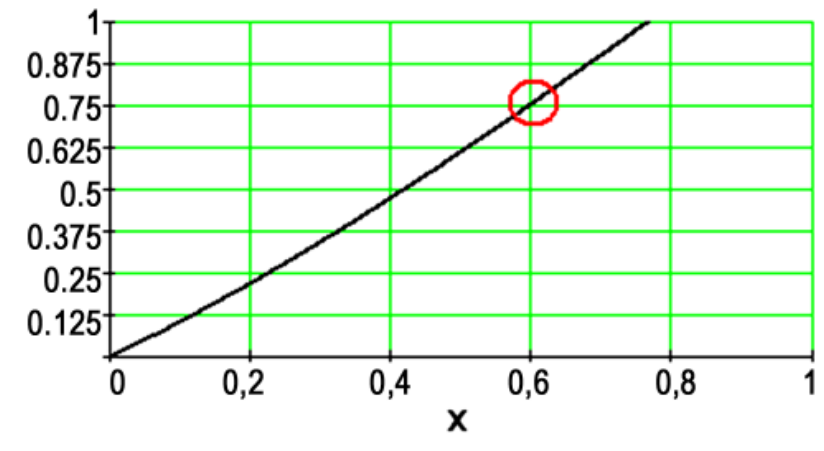

Fig. 9. Gráfica de la función solución.

No debemos finalizar el presente trabajo sin mencionar la aplicación de la Transformada de Laplace en la solución de las ecuaciones diferenciales de amplio uso en pasadas décadas, y que en la actualidad apenas aparece reflejada en nuestros programas de Cálculo[5] [8].

\section{ConClusión}

Con este trabajo se han analizado dos métodos para la solución numérica de ecuaciones diferenciales de primer orden, desde el punto de vista histórico y analítico, sirva pues al estudiante de Cálculo como complemento en el estudio de esta disciplina.

\section{REFERENCIAS}

[1] F. Aires, Ordinary Differential Equations. London: Addison Wesley Publishing Company, 1962.

[2] M. Spiegel, Ecuaciones diferenciales aplicadas. México: Prentice -Hall Hispanoamericana, 1983.

[3] L. Kells, Ecuaciones diferenciales elementales. Madrid: Ediciones del Castillo, 1976.

[4] H. B. Phillips, Ecuaciones diferenciales. México: UTEHA, 1945.

[5] D. Zill, Differential equations with modeling applications. México: International Thomson Editores, 2002.

[6] G. Forsythe, Computer Solution of Linear Algebraic Systems. New York: Prentece Hall, 2003.

[7] M. Morris and M. Orley, Ecuaciones Diferenciales. Madrid: Editorial Aguiar, 1972.

[8] W. Kaplan, Ordinary Differential Equations. London: Addison Wesley Publishing Company, 1958.

[9] L. Ford, Differential Equations. México: UTHEA, 1978.

[10] J. Newman, El mundo de las Matemáticas. Barcelona: Ediciones Grijalbo S.A, 1968.

[11] N.I Danilina, Matemática de Cálculo. Moscú: Editorial MIR, 1990.

[12] D. McCraken, Programación Algol y Fortran. México: Editorial Limusa, 1974.

[13] Mathcad 2001, "Manual de usuario conciso.» Reverte, Madrid, 2002. 
